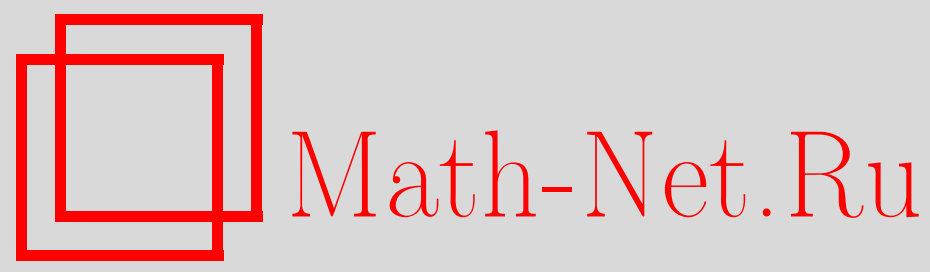

О. В. Пугачев, Пространство простых конфигураций является польским, Матем. заметки, 2002, том 71, выпуск $4,581-589$

DOI: https://doi.org/10.4213/mzm369

Использование Общероссийского математического портала Math-Net.Ru подразумевает, что вы прочитали и согласны с пользовательским соглашением http://www.mathnet.ru/rus/agreement

Параметры загрузки:

IP: 18.208 .226 .222

26 апреля 2023 г., 12:24:03 


\title{
ПРОСТРАНСТВО ПРОСТЫХ КОНФИГУРАЦИЙ ЯВЛЯЕТСЯ ПОЛЬСКИМ
}

\section{О.В. Пугачев}

\begin{abstract}
В работе доказьвается, что пространство конфигураций без кратных точек на гладком римановом многообразии, наделенное слабой топологией, является польским; приводится критерий предкомпактности множества в этом пространстве.

Библиограбой: 13 названий.
\end{abstract}

В 1990-егоды возрос интерес к анализу в пространствах конфигураций на римановых многообразиях (см., например, [1]-[7]). Пространства конфигураций представляют собой интересньй пример бесконечномерных многообразий, относительно простых в описании и обладающих разнообразными аналитическими и геометрическими свойствами. Меры на этих пространствах также представляют большой интерес (см. [8]-[10]).

Есть два подхода к описанию пространства конфигураций: можно допускать конфигурации с кратными точками или же рассматривать только простые конфигурации, т.е. не имеющие кратных точек. Пространство конфигураций с кратными точками обладает болеепростьми геометрическими и топологическими свойствами. В частности, известно, что оно является польским со слабой топологией (см. [4]), т.е. его слабая топология задается метрикой, относительно которой оно полно и сепарабельно. В то же время пространство без кратных точек оказьвается более удобным для физических приложений (поскольку в одной точке не могут находиться одновременно несколько частиц), и на нем удобно строить диффузионные процессы (см. [11]).

Цель данной работы - доказать, что пространство конфигураций без кратных точек также является польским (это свойство весьма важно в теории мер, см. [12, гл. 1]). Кроме того, вьводится критерий предкомпактности множества в этом пространстве.

1. Топология и метрика пространства конфигураций. Пусть $M$ - некомпактное связное полное локально компактное метрическое пространство (например, риманово многообразие конечной размерности). Через $\operatorname{dist}_{M}(x, y)$ будем обозначать расстояние между точками $x, y \in M$ в полной метрике на $M$, в которой всякое ограниченное множество предкомпактно. Обозначим через $C_{0}(M)$ класс непрерывных функций на $M$ с компактными носителями, а через $C_{0}(U)$ будем обозначать класс функций $\varphi \in C_{0}(M)$ таких, что $\operatorname{supp} \varphi \subset \operatorname{Int} U, U \subset M$.

Если $A \subset M$, то через $1_{A}$ будем обозначать индикаторную функцию:

$$
1_{A}(x)= \begin{cases}1, & \text { если } x \in A, \\ 0, & \text { если } x \notin A .\end{cases}
$$


ОПРЕДЕЛЕНИЕ 1.1. Пространство $\widehat{\Gamma}$ конфигураций с кратными точками на $M$ состоит из натуральнозначных $\sigma$-конечных мер $\gamma$ на $M$ таких, что $\gamma(A)<\infty$ для любого компакта $A \subset M$. Иначе говоря, $\gamma$ имеет вид

$$
\gamma=\sum_{i=1}^{N} k_{i} \delta_{x_{i}}, \quad N \in \mathbb{N} \cup\{+\infty\}, \quad x_{i} \in M, \quad k_{i} \in \mathbb{N},
$$

где $k_{i}-$ кратность точки $x_{i}, \delta_{x_{i}}$ - мера Дирака, сосредоточенная в $x_{i}$, и последовательность $\left\{x_{i}\right\}_{i=1}^{\infty}$ не имеет предельных точек.

ОПРЕДЕЛЕНИЕ 1.2. Слабой топологией на $\widehat{\Gamma}$ будем назьвать топологию $\sigma$, порожденную функциями вида

$$
\langle\varphi, \gamma\rangle=\int \varphi(x) \gamma(d x), \quad \varphi \in C_{0}(M)
$$

т.е. стандартные окрестности в этой топологии имеют вид

$$
U=\left\{\gamma:\left|\left\langle\varphi_{j}, \gamma-\gamma_{o}\right\rangle\right|<\varepsilon, j=1, \ldots, m\right\}, \quad m \in \mathbb{N}, \quad \varphi_{j} \in C_{0}(M) .
$$

Известно, что пространство $\widehat{\Gamma}$ со слабой топологией является польским, т.е. гомеоморфно полному сепарабельному метрическому пространству. Для удобства дальнейших рассуждений приведем доказательство этого факта.

Зафиксируем точку $о \in M$. Будем обозначать

$$
B_{r}=\left\{x \in M: \operatorname{dist}_{M}(o, x) \leqslant r\right\} .
$$

Для любых $R>r>0$ существует функция $\theta_{r}^{R} \in C_{0}(M)$ такая, что $1_{B_{r}} \leqslant \theta_{r}^{R} \leqslant 1_{B_{R}}$.

Лемма 1.3. Слабая топология на $\widehat{\Gamma}$ порождается счетным набором функиий вида (1).

ДокАЗАТЕЛЬСТво. При каждом $R>0$ класс функций $C_{0}\left(B_{R}\right)$ является сепарабельным относительно равномерной сходимости, следовательно, существуют функции $\psi_{R, 1}, \psi_{R, 2}, \ldots \in C_{0}\left(B_{R}\right)$ такие, что любая функция $f \in C_{0}\left(B_{R}\right)$ равномерно приближается ими на $B_{R}$. Продолжим эти функции на все $M$, положив $\psi_{R, j}=0$ вне $B_{R}$.

Возьмем счетный набор функций

$$
\left\{\theta_{n}^{n+1}\right\}_{n \in \mathbb{N}} \cup\left\{\psi_{n, k}\right\}_{n, k \in \mathbb{N}} .
$$

Тогда топология $\tau$, порожденная функциями $\left\langle\theta_{n}^{n+1}, \gamma\right\rangle$ и $\left\langle\psi_{n, k}, \gamma\right\rangle$, совпадает со слабой топологией $\sigma$. Достаточно доказать, что $\tau$ не слабее $\sigma$, т.е. что в любой $\sigma$-окрестности любой конфигурации $\gamma_{o}$ содержится $\tau$-окрестность $\gamma_{o}$. Пусть

$$
U=\left\{\gamma:\left|\left\langle\varphi_{j}, \gamma-\gamma_{o}\right\rangle\right|<1, j=1, \ldots, m\right\}, \quad \varphi_{j} \in C_{0}(M) .
$$

Выберем такое $n$, что $\operatorname{supp} \varphi_{j} \subset B_{n}, j=1, \ldots, m$. Для каждого номера $j$ найдем функцию $\psi_{n, k_{j}}$ такую, что

$$
\sup _{x \in B_{n}}\left|\psi_{n, k_{j}}(x)-\varphi_{j}(x)\right|<\frac{1 / 3}{\left\langle\theta_{n}^{n+1}, \gamma_{o}\right\rangle+1} .
$$


Возьмем $\tau$-окрестность $\gamma_{o}$ вида

$$
U_{0}=\left\{\gamma:\left|\left\langle\theta_{n}^{n+1}, \gamma-\gamma_{o}\right\rangle\right|<1\right\} \cap\left\{\left|\left\langle\psi_{n, k_{j}}, \gamma-\gamma_{o}\right\rangle\right|<\frac{1}{3}, j=1, \ldots, m\right\} .
$$

Тогда для каждой $\gamma \in U_{0}$ и $j=1, \ldots, m$ мы имеем

$$
\begin{aligned}
\left|\left\langle\varphi_{j}, \gamma-\gamma_{o}\right\rangle\right| & \leqslant\left|\left\langle\psi_{n, k_{j}}, \gamma-\gamma_{o}\right\rangle\right|+\left\langle\left|\varphi_{j}-\psi_{n, k_{j}}\right|, \gamma_{o}\right\rangle+\left\langle\left|\varphi_{j}-\psi_{n, k_{j}}\right|, \gamma\right\rangle \\
& <\frac{1}{3}+\frac{1 / 3}{\left\langle\theta_{n}^{n+1}, \gamma_{o}\right\rangle+1}\left(\gamma_{o}\left(B_{n}\right)+\gamma\left(B_{n}\right)\right) \\
& \leqslant \frac{1}{3}+\frac{1 / 3}{\left\langle\theta_{n}^{n+1}, \gamma_{o}\right\rangle+1}\left(\left\langle\theta_{n}^{n+1}, \gamma_{o}\right\rangle+\left\langle\theta_{n}^{n+1}, \gamma\right\rangle\right)<1 .
\end{aligned}
$$

Следовательно, $U_{0} \subset U$.

СледСТвиЕ 1.4. Существует метрика $\rho$, задающая топологию $\sigma=\tau$.

ДокАЗАТЕльСтво. Перенумеруем функции, задающие топологию $\tau$ :

$$
\left\{\varphi_{i}\right\}_{i=1}^{\infty}=\left\{\theta_{n}^{n+1}\right\}_{n \in \mathbb{N}} \cup\left\{\psi_{n, k}\right\}_{n, k \in \mathbb{N}} .
$$

Положим

$$
\rho(\gamma, \zeta)=\sum_{i=1}^{\infty} \min \left\{2^{-i} ;\left|\left\langle\varphi_{i}, \gamma-\zeta\right\rangle\right|\right\}
$$

Легко проверить, что $\rho$ является метрикой и задает сходимость в топологии $\tau$.

ТЕОРема 1.5. Пространство $\widehat{\Gamma}$ с метрикой $\rho$ является полныц и сепарабельHbL.M.

ДоказАТЕЛЬСтво. (i) Пусть $\left\{\gamma_{k}\right\}_{k=1}^{\infty}$ - фундаментальная последовательность в $(\widehat{\Gamma}, \rho)$. Для любых $R>r>0$ существует $\lim _{k \rightarrow \infty}\left\langle\theta_{r}^{R}, \gamma_{k}\right\rangle=: M_{r}^{R}$. Зафиксируем $n \in \mathbb{N}$. Очевидно, что $M_{n}^{n+1} \leqslant M_{n+2}^{n+3}$.

(а) Если $M_{n+2}^{n+3}-M_{n}^{n+1}<1$, то найдется единственное целое число $N_{n}$ такое, что $M_{n}^{n+1} \leqslant N_{n} \leqslant M_{n+2}^{n+3}$, и поскольку

$$
\left\langle\theta_{n}^{n+1}, \gamma_{k}\right\rangle \leqslant \gamma_{k}\left(B_{n+1}\right) \leqslant\left\langle\theta_{n+2}^{n+3}, \gamma_{k}\right\rangle \quad \forall k,
$$

то сушествует такой номер $K_{n}$, что $\gamma_{k}\left(B_{n+1}\right)=N_{n}$ при $k \geqslant K_{n}$. Положим $R_{n}:=n+1$.

(b) Если $M_{n+2}^{n+3}-M_{n}^{n+1} \geqslant 1$, то выберем $l>M_{n+2}^{n+3}-M_{n}^{n+1}, l \in \mathbb{N}$, и рассмотрим следуюшие $l+2$ функций из класса $C_{0}(M)$ :

$$
\begin{aligned}
& \varphi_{0}:=\theta_{n}^{n+1} ; \\
& \varphi_{j}:=\theta_{n+1+(j)}^{n+1+1) / l}, j=1, \ldots, l ; \\
& \varphi_{l+1}:=\theta_{n+2}^{n+3} .
\end{aligned}
$$

Очевидно, что $\varphi_{j} \leqslant 1_{B_{n+1+j / l}} \leqslant \varphi_{j+1}$. Тогда найдется такое $j, 0 \leqslant j \leqslant l$, что

$$
\lim _{k \rightarrow \infty}\left\langle\varphi_{j+1}, \gamma_{k}\right\rangle-\lim _{k \rightarrow \infty}\left\langle\varphi_{j}, \gamma_{k}\right\rangle<1,
$$

и, повторив рассуждения из пункта (а), найдем $R_{n}:=n+1+j / l$ и целое

$$
N_{n} \in\left[\lim _{k \rightarrow \infty}\left\langle\varphi_{j}, \gamma_{k}\right\rangle ; \lim _{k \rightarrow \infty}\left\langle\varphi_{j+1}, \gamma_{k}\right\rangle\right]
$$


такие, что с некоторьм $K_{n}$ для всех $k \geqslant K_{n}$ вьполнено равенство $\gamma_{k}\left(B_{R_{n}}\right)=N_{n}$.

Поскольку это рассуждение проходит для всех $n \in \mathbb{N}$, мы получаем последовательность шаров

$$
B_{R_{1}} \subset B_{R_{2}} \subset \cdots, \quad R_{n} \rightarrow \infty
$$

таких, что

$$
\exists K_{n}: \quad \forall k \geqslant K_{n} \gamma_{k}\left(B_{R_{n}}\right)=N_{n} .
$$

При каждом $R_{n}$ локальные конфигурации $\left.\gamma_{k}\right|_{B_{R_{n}}}, k \geqslant K_{n}$, можно рассматривать как точки компакта $B_{R_{n}}^{N_{n}} / S_{N_{n}}$, где $S_{N_{n}}$ обозначает группу перестановок $N_{n}$ элементов. Из любой подпоследовательности $\left\{\left.\gamma_{k_{i}}\right|_{B_{R_{n}}}\right\}_{i=1}^{\infty}$ можновыбрать подпоследовательность $\left\{\left.\gamma_{k_{j}}\right|_{B_{R_{n}}}\right\}_{j=1}^{\infty}$, сходящуюся на $B_{R_{n}}^{N_{n}} / S_{N_{n}}$ к некоторой $N_{n}$-точечной (точки всегда считаются с учетом кратности) конфигурации $\gamma^{(n)}$ на $B_{R_{n}}$. При этом разныеподпоследовательности будут давать одну и ту же предельную конфигурацию $\gamma^{(n)}$. Действительно, если $\left.\gamma_{i}^{*}\right|_{B_{R_{n}}} \rightarrow \gamma^{(n)},\left.\gamma_{i}^{* *}\right|_{B_{R_{n}}} \rightarrow \zeta$ и $\zeta \neq \gamma^{(n)}$, то найдется функция $\varphi \in C_{0}(M)$ с носителем на $B_{R_{n}}$ такая, что $\left\langle\varphi, \gamma^{(n)}\right\rangle \neq\langle\varphi, \zeta\rangle$, откуда

$$
\lim _{i \rightarrow \infty}\left\langle\varphi, \gamma_{i}^{*}\right\rangle \neq \lim _{i \rightarrow \infty}\left\langle\varphi, \gamma_{i}^{* *}\right\rangle
$$

что противоречит фундаментальности $\left\{\gamma_{k}\right\}$ по $\rho$, поскольку функция $\langle\varphi, \cdot\rangle$ непрерывна по $\rho$. Отсюда следует, что вся последовательность $\left\{\left.\gamma_{k}\right|_{B_{R_{n}}}\right\}_{k=K_{n}}^{\infty}$ сходится к $\gamma^{(n)}$. Очевидно, что при $m>n \gamma^{(m)} \geqslant \gamma^{(n)}$. Возьмем предел возрастающих мер

$$
\gamma_{o}:=\lim _{n \rightarrow \infty} \gamma^{(n)}
$$

Мера $\gamma_{o}$ является $\sigma$-конечной и принимает натуральные значения; ее носитель не имеет предельных точек, так как любой компакт $A \subset M$ вложен в некоторьй шар $B_{R_{n}}$, на котором $\gamma_{o}$ конечна. Следовательно, $\gamma_{o} \in \widehat{\Gamma}$. Легко проверить, что $\left\langle\varphi, \gamma_{k}\right\rangle \rightarrow\left\langle\varphi, \gamma_{o}\right\rangle$ при всех $\varphi \in C_{0}(M)$.

(ii) Пусть $S$ - счетное плотное подмножество $M$. Рассмотрим счетное множество $\mathscr{S}$ конечных конфигураций на $S$. Зафиксируем $\gamma_{o} \in \widehat{\Gamma}$ и функции $\varphi_{j}, j=1, \ldots, m$, задающие окрестность вида (2). Пусть

$$
\Omega:=\bigcup_{j=1}^{m} \operatorname{supp} \varphi_{j}, \quad N:=\gamma_{o}(\Omega) .
$$

Пусть $\delta<\varepsilon\left(N \sup _{1 \leqslant j \leqslant m}\left|\nabla \varphi_{j}\right|\right)^{-1}$. Можно найти $N$-точечную конфигурацию $\gamma^{\prime}$ на $\Omega \cap S$ такую, что

$$
\forall x \in \operatorname{supp} \gamma_{o} \cap \Omega \quad \exists x^{\prime} \in \operatorname{supp} \gamma^{\prime}: \quad \operatorname{dist}_{M}\left(x, x^{\prime}\right)<\delta
$$

и кратности точек $x$ в $\gamma_{o}$ и $x^{\prime}$ в $\gamma^{\prime}$ совпадают. Тогда

$$
\left|\left\langle\varphi_{j}, \gamma^{\prime}-\gamma_{o}\right\rangle\right|<\delta N \sup _{1 \leqslant j \leqslant m}\left|\nabla \varphi_{j}\right|<\varepsilon .
$$


ОПРЕДЕЛЕНИЕ 1.6. Пространство Г простых конфигураций $M$ есть подмножество $\widehat{\Gamma}$, задаваемое условием

$$
\Gamma=\{\gamma \in \widehat{\Gamma}: \quad \forall x \in M \quad \gamma(\{x\}) \leqslant 1\} .
$$

На Г будем рассматривать ту же слабую топологию $\sigma$, которая по-прежнему может быть задана метрикой $\rho$. Но $(\Gamma, \rho)$ уже не является полным метрическим пространством, поскольку сушествует $\rho$-фундаментальная последовательность конфигураций из $\Gamma$, сходящаяся к конфигурации с кратной точкой. Однако пространство может быть полньп по одной метрике и не полным по другой, даже если обе метрики задают одну и ту же топологию.

ПРИмеР. Пусть $X=(-\pi / 2 ; \pi / 2)$. Рассмотрим две метрики, задающие обычную сходимость:

$$
d_{1}(x, y)=|x-y| ; \quad d_{2}(x, y)=|\operatorname{tg} x-\operatorname{tg} y| .
$$

Относительно $d_{1}$ пространство $X$ неполно, а относительно $d_{2}$ оно полно, поскольку функция $\operatorname{tg}$ осуществляет его изометрическую биекцию на пространство $\mathbb{R}$, наделенное метрикой $d_{1}$.

Докажем, что пространство Г со слабой топологией также является польским. Введем на Г функции

$$
h_{n}(\gamma)=\sum_{x, y \in \operatorname{supp} \gamma, x \neq y} \frac{\theta_{n}^{n+1}(x) \theta_{n}^{n+1}(y)}{\operatorname{dist}_{M}(x, y)}
$$

Эта величина конечна, так как $\gamma$ имеет конечное число точек на $\operatorname{supp} \theta_{n}^{n+1} \subset B_{n+1}$.

Лемма 1.7. (i) Функиия $h_{n}$ слабо непрерывна на Г.

(ii) Если последовательность конфигураций $\left\{\gamma_{k}\right\}$ слабо сходится $\kappa \gamma_{o} \in \widehat{\Gamma} \backslash \Gamma$, причем $\gamma_{о}$ имеет кратную точку в $B_{n}$, mо $h_{n}\left(\gamma_{k}\right) \rightarrow+\infty$.

ДокАЗАтЕЛЬСтво. (i) Зафиксируем $\gamma_{o} \in \Gamma$. Пусть

$$
l:=\min _{x, y \in B_{n+1} \cap \operatorname{supp} \gamma_{o}} \operatorname{dist}_{M}(x, y), \quad N:=\gamma_{o}\left(B_{n+1}\right),
$$

$\operatorname{dist}_{M}\left(\operatorname{supp} \gamma_{o} \backslash B_{n+1}, B_{n+1}\right)=: 2 \delta$. Поскольку в $B_{n+2}$ лежит конечное число точек $\gamma_{o}$, мы имеем $\delta>0$. Возьмем $\varepsilon \in(0 ; \min \{\delta, l / 3\})$. Зафиксируем функции

$$
\varphi_{\varepsilon}^{y} \in C_{0}(M), \quad 1_{\{y\}} \leqslant \varphi_{\varepsilon}^{y} \leqslant 1_{\left\{\operatorname{dist}_{M}(y, \cdot)<\varepsilon\right\}},
$$

и рассмотрим функцию

$$
\varphi_{\varepsilon}=\sum_{y \in B_{n+1} \cap \operatorname{supp} \gamma_{o}} \varphi_{\varepsilon}^{y} .
$$

Тогда $\left\langle\varphi_{\varepsilon}^{y}, \gamma_{o}\right\rangle=1,\left\langle\varphi_{\varepsilon}, \gamma_{o}\right\rangle=N$. Если

$$
\begin{aligned}
\gamma \in U_{\varepsilon}:= & \left\{\left|\left\langle\varphi_{\varepsilon}, \gamma\right\rangle-N\right|<\frac{1}{2}, \quad\left|\left\langle\theta_{n+1+\delta}^{n+1+2 \delta}, \gamma\right\rangle-N\right|<\frac{1}{2}\right\} \\
& \cap\left\{\left|\left\langle\varphi_{\varepsilon}^{y}, \gamma\right\rangle-1\right|<\frac{1}{2} \quad \forall y \in B_{n+1} \cap \operatorname{supp} \gamma_{o}\right\},
\end{aligned}
$$

то $\gamma\left(B_{n+1+\delta}\right)=N$ и при этом

$$
\forall x \in \operatorname{supp} \gamma_{o} \cap B_{n+1} \quad \exists ! x^{\prime} \in \operatorname{supp} \gamma \cap B_{n+1+\delta}: \quad \operatorname{dist}_{M}\left(x, x^{\prime}\right)<\varepsilon .
$$


Для любых $x, y \in \operatorname{supp} \gamma_{o} \cap B_{n+1}$ и соответствуюших им $x^{\prime}, y^{\prime} \in \operatorname{supp} \gamma$ мы имеем

$$
\begin{aligned}
& \left|\frac{\theta_{n}^{n+1}\left(x^{\prime}\right) \theta_{n}^{n+1}\left(y^{\prime}\right)}{\operatorname{dist}_{M}\left(x^{\prime}, y^{\prime}\right)}-\frac{\theta_{n}^{n+1}(x) \theta_{n}^{n+1}(y)}{\operatorname{dist}_{M}(x, y)}\right| \\
& \quad<\frac{\left|\theta_{n}^{n+1}\left(x^{\prime}\right)-\theta_{n}^{n+1}(x)\right|+\left|\theta_{n}^{n+1}\left(y^{\prime}\right)-\theta_{n}^{n+1}(y)\right|}{l-2 \varepsilon}+\left(\frac{1}{l-2 \varepsilon}-\frac{1}{l}\right) \\
& \quad \leqslant \frac{2 \sup \left|\nabla \theta_{n}^{n+1}\right| \varepsilon}{l / 3}+\frac{2 \varepsilon}{l(l / 3)}=C\left(\gamma_{o}, n\right) \varepsilon .
\end{aligned}
$$

Следовательно,

$$
\left|h_{n}(\gamma)-h_{n}\left(\gamma_{o}\right)\right|<\frac{N(N-1)}{2} C\left(\gamma_{o}, n\right) \varepsilon
$$

Построив окрестности $U_{\varepsilon}$ при сколь угодно малых $\varepsilon>0$, получаем непрерьвность $h_{n}$ в $\gamma_{o}$.

(ii) Пусть последовательность $\left\{\gamma_{k}\right\}$ слабо сходится к $\gamma_{o} \in \widehat{\Gamma} \backslash \Gamma$; пусть $x_{o} \in B_{n}-$ кратная точка $\gamma_{o}$, т.е. $\gamma_{o}\left(\left\{x_{o}\right\}\right) \geqslant 2$. При сколь угодно малом $\varepsilon>0$ таком, что

$$
\sup _{B_{n+\varepsilon}} \theta_{n}^{n+1} \geqslant \frac{1}{2}
$$

возьмем функцию $\varphi_{\varepsilon}^{x_{o}}$ вида $(3)$; тогда $\left\langle\varphi_{\varepsilon}^{x_{o}}, \gamma_{k}\right\rangle \rightarrow\left\langle\varphi_{\varepsilon}^{x_{o}}, \gamma_{o}\right\rangle \geqslant 2$, откуда следует, что при всех $k$, начиная с некоторого $K(\varepsilon)$, вьполняется оценка

$$
\gamma_{k}\left\{\operatorname{dist}_{M}\left(x, x_{o}\right) \leqslant \varepsilon\right\} \geqslant\left\langle\varphi_{\varepsilon}^{x_{o}}, \gamma_{k}\right\rangle>3 / 2,
$$

следовательно, $\gamma_{k}\left\{\operatorname{dist}_{M}\left(x, x_{o}\right) \leqslant \varepsilon\right\} \geqslant 2$, т.е. $\varepsilon$-окрестность точки $x_{o}$ содержит хотя бы две точки $x$ и $y$ конфигурации $\gamma_{k}$ и, поскольку эти точки лежат в шаре $B_{n+\varepsilon}$, получаем оценку

$$
h_{n}\left(\gamma_{k}\right) \geqslant \frac{\theta_{n}^{n+1}(x) \theta_{n}^{n+1}(y)}{\operatorname{dist}_{M}(x, y)} \geqslant \frac{(1 / 2)(1 / 2)}{2 \varepsilon}=\frac{1}{8 \varepsilon} .
$$

ТЕОРема 1.8. На пространстве Г существует метрика $\rho_{1}$ со следующими свойствами:

(i) метрика $\rho_{1}$ задает топологию $\sigma$;

(ii) пространство $\left(\Gamma, \rho_{1}\right)$ полно;

(iii) пространство $\left(\Gamma, \rho_{1}\right)$ сепарабельно.

ДокАЗАТЕЛьство. Введем на Г метрику

$$
\rho_{1}(\gamma, \zeta):=\rho(\gamma, \zeta)+\sum_{n=1}^{\infty} \min \left\{2^{-n},\left|h_{n}(\gamma)-h_{n}(\zeta)\right|\right\}
$$

(i) Докажем, что топология, задаваемая метрикой $\rho_{1}$, не сильнее слабой топологии (задаваемой метрикой $\rho$ ). Пусть $\rho\left(\gamma_{m}, \gamma_{o}\right) \rightarrow 0, \gamma_{m}, \gamma_{o} \in \Gamma$. Поскольку функции $h_{n}$ непрерьвны по $\rho$ на $\Gamma$, мы получаем $\left|h_{n}\left(\gamma_{m}\right)-h_{n}\left(\gamma_{o}\right)\right| \rightarrow 0$ при $m \rightarrow \infty$ для любого $n \in \mathbb{N}$, откуда следует $\rho_{1}\left(\gamma_{m}, \gamma_{o}\right) \rightarrow 0$.

(ii) Пусть $\gamma_{m} \in \Gamma$ и последовательность $\left\{\gamma_{m}\right\}_{m=1}^{\infty}$ фундаментальна по $\rho_{1}$. Тогда $\left\{\gamma_{m}\right\}_{m=1}^{\infty}$ фундаментальна и по $\rho$, и в силу полноты $(\widehat{\Gamma}, \rho)$ существует конфигурация $\gamma_{o} \in \widehat{\Gamma}$ такая, что $\rho\left(\gamma_{m}, \gamma_{o}\right) \rightarrow 0$. Если $\gamma_{o} \in \Gamma$, то в силу пункта (i) мы получим 
$\rho_{1}\left(\gamma_{m}, \gamma_{o}\right) \rightarrow 0$, что и требовалось доказать. Если же $\gamma_{o} \notin \Gamma$, то в силу пункта (ii) леммы 1.7 существует $n \in \mathbb{N}$, при котором $h_{n}\left(\gamma_{m}\right) \rightarrow+\infty$, откуда вытекает, что существует подпоследовательность $\left\{\gamma_{m_{i}}\right\}$ такая, что $\left|h_{n}\left(\gamma_{m_{i+1}}\right)-h_{n}\left(\gamma_{m_{i}}\right)\right|>1$. Следовательно, $\rho_{1}\left(\gamma_{m_{i+1}}, \gamma_{m_{i}}\right) \geqslant 2^{-n}$, что противоречит предположению о фундаментальности $\left\{\gamma_{m}\right\}$ по $\rho_{1}$.

(iii) Пусть $S$ - счетное плотное подмножество $M$. Рассмотрим счетное множество $\mathscr{S}_{1}$ конечных конфигураций на $S$ без кратных точек. Зафиксируем $\gamma_{o} \in \Gamma, n \in \mathbb{N}$. Для любого $\varepsilon>0$ можно найти конечную конфигурацию $\gamma_{n}^{\varepsilon}$ на $S \cap B_{n}$ без кратньх точек такую, что

$$
\forall x \in \operatorname{supp} \gamma_{o} \cap B_{n} \quad \exists ! x^{\prime} \in \operatorname{supp} \gamma_{n}^{\varepsilon}: \quad \operatorname{dist}_{M}\left(x, x^{\prime}\right)<\varepsilon .
$$

Построим последовательность конфигураций $\left\{\gamma_{n}^{1 / n}\right\}_{n=1}^{\infty} \subset \mathscr{S}_{1}$. Докажем, что они слабо сходятся к $\gamma_{о}$ (следовательно, по доказанному пункту (i) они сходятся и по метрике $\left.\rho_{1}\right)$. Действительно, пусть $\varphi \in C_{0}(M), \operatorname{supp} \varphi \subset B_{n}, \sup |\nabla \varphi|=c, \gamma_{o}\left(B_{n}\right)=N$. Тогда при $m \geqslant n$ мы имеем

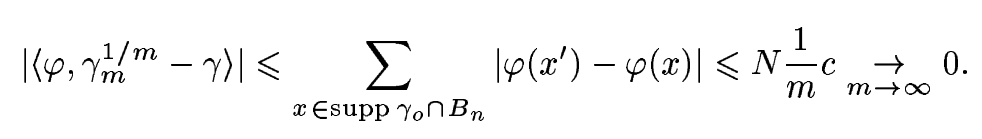

\section{2. Компактность в пространстве конфигураций.}

ТЕорема 2.1. Чтобы подмножсество $Q$ пространства $\widehat{\Gamma}$ было предкомпактным в слабой топологии, необходимо и достаточно выполнения следуюиего условия: для любого компакта $A \subset M$

$$
\sup _{\gamma \in Q} \gamma(A)<\infty
$$

ДокАЗАтЕЛЬСтво. Необходимость. Пусть $A$ - компактное подмножество $M$. Существует функция $\varphi \in C_{0}(M)$ такая, что $\varphi \geqslant 1_{A}$. Поскольку функция $\langle\varphi, \cdot\rangle$ слабо непрерьвна на $\widehat{\Gamma}$, она ограничена на $Q$. Следовательно,

$$
\sup _{\gamma \in Q} \gamma(A) \leqslant \sup _{\gamma \in Q}\langle\varphi, \gamma\rangle<\infty
$$

Достаточность. Пусть (4) вьполнено хотя бы для шаров $B_{1}, B_{2}, B_{3}, \ldots$ Рассмотрим множество

$$
K=\left\{\gamma \in \widehat{\Gamma}: \quad \gamma\left(B_{n}\right) \leqslant N_{n} \quad \forall n \in \mathbb{N}\right\}
$$

где $N_{n}=\sup _{\gamma \in Q} \gamma\left(B_{n}\right)$. Тогда $K \supset Q$. Докажем, что $K-$ компакт в слабой топологии. Пусть $\left\{\gamma_{j}\right\}_{j=1}^{\infty}-$ последовательность конфигураций из $K$. Поскольку $\left\{\gamma_{j}\left(B_{1}\right)\right\}_{j=1}^{\infty}$ принимает лиш значения $0,1, \ldots, N_{1}$, хотя бы одно из этих значений, скажем $k_{1}$, встречается бесконечно много раз. Среди тех $\gamma_{j}$, при которых $\gamma_{j}\left(B_{1}\right)=k_{1}$, выберем подпоследовательность $\left\{\gamma_{i}^{1}\right\}_{i=1}^{\infty}$ такую, что локальные конфигурации $\left.\gamma_{i}^{1}\right|_{B_{1}}$ сходятся к некоторой $k_{1}$-точечной конфигурации $\gamma^{1}$ на $B_{1}$.

Повторяя эту процедуру для шаров $B_{2}, B_{3}, \ldots$, построим подпоследовательности

$$
\left\{\gamma_{i}^{1}\right\}_{i=1}^{\infty} \supset\left\{\gamma_{l}^{2}\right\}_{l=1}^{\infty} \supset\left\{\gamma_{m}^{3}\right\}_{m=1}^{\infty} \supset \cdots
$$


такие, что $\left\{\left.\gamma_{m}^{n}\right|_{B_{n}}\right\}_{m=1}^{\infty}$ слабо сходится к некоторой конфигурации $\gamma^{n}$ на $B_{n}$. Очевидно, что $\gamma_{n} \leqslant \gamma_{n+1}$. Наконец, выберем диагональную подпоследовательность $\left\{\gamma_{n}^{n}\right\}_{n=1}^{\infty}$. Она слабо сходится к конфигурации $\gamma_{o}=\sup _{n \in \mathbb{N}} \gamma^{n} \in K$ : действительно, если $\varphi \in C_{0}(M)$, то найдется $n$ такое, что $\operatorname{supp} \varphi \subset B_{n}$ и при $m \geqslant n$

$$
\left\langle\varphi, \gamma_{m}^{m}\right\rangle=\left\langle\varphi,\left.\gamma_{m}^{m}\right|_{B_{n}}\right\rangle \underset{m \rightarrow \infty}{\rightarrow}\left\langle\varphi, \gamma^{n}\right\rangle=\left\langle\varphi, \gamma_{o}\right\rangle \text {. }
$$

Таким образом, множество $K$ компактно.

ТЕОРема 2.2. Чтобы подмножсество $Q$ пространства Г было предкомпактным в слабой топологии, необходимо и достаточно выполнения условия: для любого компакта $A \subset M$ выполнены (4) $и$

$$
\inf _{\gamma \in Q} \min _{x, y \in \operatorname{supp} \gamma \cap A} \operatorname{dist}_{M}(x, y)>0 .
$$

ДокАЗАтЕЛЬСтво. Необходимость. Поскольку $Q$ предкомпактно в Г по метрике $\rho_{1}$, оно имеет конечную $\varepsilon$-сеть относительно $\rho_{1}$ (следовательно, и относительно $\rho$ ) при любом $\varepsilon>0$. Следовательно, $Q$ предкомпактно в $(\widehat{\Gamma}, \rho)$, откуда в силу теоремы 2.1 вытекает (4). Теперь предположим, что нарушено условие (6) для некоторого компакта $A \subset M$. Пусть $A \subset B_{n}$. Тогда существует последовательность $\left\{\gamma_{k}\right\}_{k=1}^{\infty} \subset Q$ такая, что

$$
\forall k \quad \exists x_{k}, y_{k} \in \operatorname{supp} \gamma_{k} \cap B_{n}: \quad \operatorname{dist}_{M}\left(x_{k}, y_{k}\right)<\frac{1}{k} .
$$

Следовательно,

$$
h_{n}\left(\gamma_{k}\right) \geqslant \frac{\theta_{n}^{n+1}\left(x_{k}\right) \theta_{n}^{n+1}\left(y_{k}\right)}{\operatorname{dist}_{M}\left(x_{k}, y_{k}\right)}>k,
$$

что приводит к противоречию, так как функция $h_{n}$ слабо непрерывна на $\Gamma$ по лемме 1.7 и должна быть ограниченной на предкомпактном множестве $Q \subset \Gamma$.

Достаточность. Пусть выполнены условия (4) и (6) хотя бы для компактов $B_{n}, n=1$, $2, \ldots$. Обозначим

$$
N_{n}:=\sup _{\gamma \in Q} \gamma\left(B_{n}\right), \quad l_{n}:=\inf _{\gamma \in Q} \min _{x, y \in \operatorname{supp} \gamma \cap B_{n}} \operatorname{dist}_{M}(x, y)
$$

и рассмотрим множество

$$
K_{1}=\left\{\gamma \in \Gamma: \gamma\left(B_{n}\right) \leqslant N_{n}, \quad \min _{x, y \in \operatorname{supp} \gamma} \frac{\operatorname{dist}_{M}(x, y)}{\theta_{n}^{n+1}(x) \theta_{n}^{n+1}(y)} \geqslant l_{n} \quad \forall n \in \mathbb{N}\right\} .
$$

Очевидно, что $K_{1} \supset Q$. Докажем, что $K_{1}$ компактно в слабой топологии. Поскольку $K_{1}$ содержится в компакте $K$, заданном формулой (5), из любой последовательности $\left\{\gamma_{j}\right\}_{j=1}^{\infty} \subset K_{1}$ можно выбрать подпоследовательность $\left\{\gamma_{(i)}\right\}_{i=1}^{\infty} \subset K_{1}$, слабо сходящуюся к некоторой конфигурации $\gamma_{o} \in K$. Но если $\gamma_{o} \in K \backslash K_{1}$, то при некотором $n$ найдутся такие $x, y \in \operatorname{supp} \gamma_{o}$, что

$$
\theta_{n}^{n+1}(x) \geqslant \theta_{n}^{n+1}(y)>0 \quad \text { и } \quad\left(\theta_{n}^{n+1}(x) \theta_{n}^{n+1}(y)\right)^{-1} \operatorname{dist}_{M}(x, y)<l_{n} .
$$

Тогда найдется $\varepsilon \in\left(0 ; \operatorname{dist}_{M}(x, y) / 2\right)$, при котором справедлива следующая оценка: если $\operatorname{dist}_{M}(z, x)<\varepsilon, \operatorname{dist}_{M}(w, y)<\varepsilon$, то $\left(\theta_{n}^{n+1}(z) \theta_{n}^{n+1}(w)\right)^{-1} \operatorname{dist}_{M}(z, w)<l_{n}$. Но, используя функции $\varphi_{\varepsilon}^{x}, \varphi_{\varepsilon}^{y}$ вида (3), мы обнаружим, что существует $J \in \mathbb{N}$ такое, что при всех $i \geqslant J$ конфигурация $\gamma_{(i)}$ содержит точки $z_{i}$ и $w_{i}$ такие, что $\operatorname{dist}_{M}\left(z_{i}, x\right)<\varepsilon$, $\operatorname{dist}_{M}\left(w_{i}, y\right)<\varepsilon$, что приводит к противоречию с предположением $\gamma_{(i)} \in K_{1}$. Таким образом, $\gamma_{o} \in K_{1}$ и слабая компактность множества $K_{1}$, содержащего $\mathrm{Q}$, доказана. 
ОпредЕлЕниЕ 2.3. Неотрицательная мера $\mu$ на топологическом пространстве $X$ назьвается радоновской, если для любого множества $A \subset X$

$$
\mu(A)=\sup \{\mu(K): K \subset A, K \text { компактно }\} .
$$

ТЕОремА 2.4. Любая вероятностная мера на Г, как и на $\widehat{\Gamma}$, является радоновской относительно слабой топологии.

ДоКАЗАТЕЛЬСТво. Известно, что на польском пространстве всякая вероятностная мера является радоновской (см. [12, гл. 1, теорема 1.4] или [13, гл. I, предложение 1.5]).

Автор благодарит профессора В. И. Богачева за постановку интересной задачи.

\section{СПИСОК ЦИТИРОВАННОЙ ЛИТЕРАТУРЫ}

[1] Albeverio S., Daletskii A., Lytvynov E. Laplace operators on differential forms over configuration spaces // J. Geom. Phys. (to appear).

[2] Bichteler K., Gravereaux J. B., Jacod J. Malliavin Calculus for Processes with Jumps: Gordon \& Breach Sci. Publ., 1987.

[3] Давыдов Ю. А., Лифшиц М. А., Смородина Н. В. Локальные свойства распределений стохастических функционалов. М.: Физматлит, 1995.

[4] Röckner M. Stochastic analysis on configuration spaces: Basic ideas and recent results // New Directions in Dirichlet Forms. Studies in Advanced Math. V. 8. Providence (R.I.): Amer. Math. Soc., 1998.

[5] Röckner M., Schmuland B. A support property for infinite-dimensional interacting diffusion processes // C. R. Acad. Sci. Paris. Sér. I. 1998. V. 326. P. 359-364.

[6] Смородина Н.В. Дифференциальное исчисление в пространстве конфигураций и устойчивые меры. I // Теор. вероятн. и прилож. 1988. Т. 33. С. 522-534.

[7] Смородина Н. В. Формула Остроградского-Гаусса для пространства конфигураций // Теор. вероятн. и прилож. 1990. Т. 35. № 4. С. 725-736.

[8] Albeverio S., Kondratiev Yu. G., Röckner M. Analysis and geometry on configuration spaces // J. Funct. Anal. 1998. V. 154. № 2. P. 444-500.

[9] Albeverio S., Kondratiev Yu. G., Röckner M. Analysis and geometry on configuration spaces: the Gibbsian case // J. Funct. Anal. 1998. V. 157. №1. P. 242-291.

[10] Bogachev V. I., Pugachev O. V., Röckner M. Surface measures and tightness of capacities on Poisson space // J. Funct. Anal. (to appear).

[11] Ma Zhi-Ming, Röckner M. Construction of diffusions on configuration spaces // Osaka J. Math. 2000. V. 37. P. 273-314.

[12] Биллингсли П. Сходимость вероятностных мер. М.: Наука, 1977.

[13] Далецкий Ю. Л., Фомин С. В. Меры и дифференциальные уравнения в бесконечномерных пространствах. М.: Наука, 1983. 